\title{
Structural Order-Disorder Transitions and Phonon Conductivity of Partially Filled Skutterudites
}

\author{
Hyoungchul Kim, ${ }^{1}$ Massoud Kaviany, ${ }^{1, *}$ John C. Thomas, ${ }^{2}$ Anton Van der Ven, ${ }^{2}$ Ctirad Uher, ${ }^{3}$ and Baoling Huang ${ }^{4}$ \\ ${ }^{1}$ Department of Mechanical Engineering, The University of Michigan, Ann Arbor, Michigan 48109, USA \\ ${ }^{2}$ Department of Materials Science and Engineering, The University of Michigan, Ann Arbor, Michigan 48109, USA \\ ${ }^{3}$ Department of Physics, The University of Michigan, Ann Arbor, Michigan 48109, USA \\ ${ }^{4}$ Department of Mechanical Engineering, Hong Kong University of Science and Technology, Hong Kong, China \\ (Received 8 July 2010; revised manuscript received 20 September 2010; published 20 December 2010)
}

Filled skutterudites are high-performance thermoelectric materials and we show how their phonon conductivity is greatly influenced by the topology of the filler species. We predict ( $a b$ initio) the phase diagram of $\mathrm{Ba}_{x} \mathrm{Co}_{4} \mathrm{Sb}_{12}$ and find several stable configurations of $\mathrm{Ba}$ ordering over the intrinsic voids. The phonon conductivity predicted using molecular dynamics shows a minimum in the two-phase mixture regime, dominated by significantly reduced long-range acoustic phonon transport.

High-performance thermoelectric (TE) materials including skutterudites [1-4], metal silicides [5], complex chalcogenide compounds [6], clathrates [5,7], half-Heusler alloys [8], and oxides [9] are sought for efficient power generation. Skutterudites are particularly promising due to their robust mechanical properties [10] and allow for single or multiple filling with rare-earth and alkaline-earth metals that reduce the lattice thermal conductivity $\kappa_{L}$ $[1-4,11,12]$.

Binary skutterudites are compounds with the general formula $M X_{3}(M=\mathrm{Co}, \mathrm{Rh}, \mathrm{Ir}$ and $X=\mathrm{P}, \mathrm{As}, \mathrm{Sb})$ having a crystal structure with a bcc lattice and belonging to the space group $\operatorname{Im} 3$. The structure consists of a periodic array of trigonally distorted and tilted $M X_{6}$ octahedrons. The metal $M$ at the center is octahedrally coordinated by the pnicogen $X$ [13]. The tilt of the $M X_{6}$ octahedrons gives rise to empty spaces (icosahedral voids) that form a bodycentered sublattice. As first shown in [14], the voids are large enough and can be filled by foreign species entering as cations; however, the filler solubility limit is less than a full filling of all available voids $[4,15]$.

Binary skutterudites possess very high charge carrier mobilities and the interest in these compounds as novel TE materials for power generation followed a suggestion [16] and observation [11] that their $\kappa_{L}$ can be dramatically reduced upon filling the structural voids. Recent research has resulted in the TE figure of merit $(Z T)$ of $n$-type skutterudites approaching the value of 1.5 at $800 \mathrm{~K}$ [17]. While the presence of the filler species in the skutterudite matrix seems to be essential for achieving low $\kappa_{L}$ and hence high $Z T$, whether the filler acts as a rattling local vibration mode [1-4] or plays some other important role [7,18-20] remains controversial. So far the only insight into the role of ordered phases on the $\kappa_{L}$ of partially-filled skutterudites has been the observation in [3] that the random distribution of filler ions scatter phonons more effectively than when most voids are filled. In this letter, we address the issue of ordering of the filler species, a topic that has not yet been explored and that has a great influence on phonon scattering. To this end we examine $\mathrm{Ba}_{x} \mathrm{Co}_{4} \mathrm{Sb}_{12}$, a skutterudite with a large filling fraction limit for $\mathrm{Ba}$, using theoretical and computational treatments.

We begin with a determination of a temperaturecomposition phase diagram of $\mathrm{Ba}_{x} \mathrm{Co}_{4} \mathrm{Sb}_{12}$ by combining $a b$ initio calculations with the cluster expansion (CE) method and the Monte Carlo (MC) simulations. Thereafter, using equilibrium molecular dynamics (MD) simulations and the Green-Kubo fluctuation-dissipation linear response theory and $\kappa_{L}$ decomposition, along with the phase diagram, we predict the $\kappa_{L}$ of partially filled $\mathrm{Ba}_{x} \mathrm{Co}_{4} \mathrm{Sb}_{12}$ as a function of concentration and temperature.

A prediction of phase stability at finite temperature requires the use of statistical mechanics to account for entropic contributions to the free energy. The most important source of entropy in a partially filled skutterudite arises from configurational degrees of freedom associated with all possible ways of distributing $\mathrm{Ba}$ and vacancies over the bcc sites of the filler sublattice. A mathematical description of these configurational degrees of freedom is possible with the CE method [21]. An occupation variable $\sigma_{i}$ is specified for each $\mathrm{Ba}$ site $i$, which takes a value 1 if the site has a Baatom occupant and -1 if it is vacant. The $\mathrm{CE}$ is constructed from this description, assuming an on-lattice Hamiltonian that can be expressed exactly as a series expansion of configurational basis functions of the form

$$
E(\boldsymbol{\sigma})=\sum_{\{\alpha\}} V_{\alpha} \Gamma_{\alpha}(\boldsymbol{\sigma}), \quad \Gamma_{\alpha}(\boldsymbol{\sigma})=\prod_{i \in \alpha} \sigma_{i},
$$

where $\alpha$ denotes a cluster of $\mathrm{Ba}$ filler sites (pairs, triplets and so forth, as well as lone sites). All possible clusters of the sublattice sites are included in the sum. The $\Gamma_{\alpha}(\boldsymbol{\sigma})$ are cluster basis functions that form a complete and orthonormal basis within the Ba-vacancy configuration space [21]. The expansion coefficients $V_{\alpha}$ are referred to as effective cluster interactions (ECI) and can be determined from first principles. The CE describes the fully relaxed energy of the 
crystal as a function of Ba-vacancy order or disorder and can be viewed as a generalized Ising model with physically realistic interaction parameters.

The series in Eq. (1) can be truncated to reflect the relatively small contribution from clusters comprised of many sites or those describing long-length scales. As a result, density-functional theory (DFT) total energy calculations can be used to parameterize the ECI and determine the truncation of the series. This was done using the DFT energies of $28 \mathrm{Ba}$-vacancy configurations over the filler sites of $\mathrm{CoSb}_{3}$ [22]. The DFT calculations were performed using the Vienna ab initio simulation package [23] within the Perdew-Burke-Ernzerhof (PBE) parameterization of the generalized gradient approximation (GGA) for exchange and correlation [24] and using the projector augmented wave (PAW) method [25,26]. The optimized $\mathrm{CE}$ includes $10 \mathrm{ECI}$ and has a cross validation score of $6 \mathrm{meV} / \mathrm{site}$ and a rms error of $4 \mathrm{meV} /$ site.

By applying MC simulations to the cluster expanded Hamiltonian, it is possible to construct a temperaturecomposition phase diagram. The calculated phase diagram shown in Fig. 1(a) exhibits prominent ordered phases at Ba compositions below or near the experimental filling limit $(x \approx 0.44)$ [27]; the $\gamma$ phase has $x=0.25$ and the $\alpha$ phase has $x=0.5$. As shown in Figs. 1(b) and 1(c), the $\gamma$ phase is highly anisotropic with $\mathrm{Ba}-\mathrm{Ba}$ nearest neighbors (NN) along [111] and Ba-vacancy NN pairs along the other
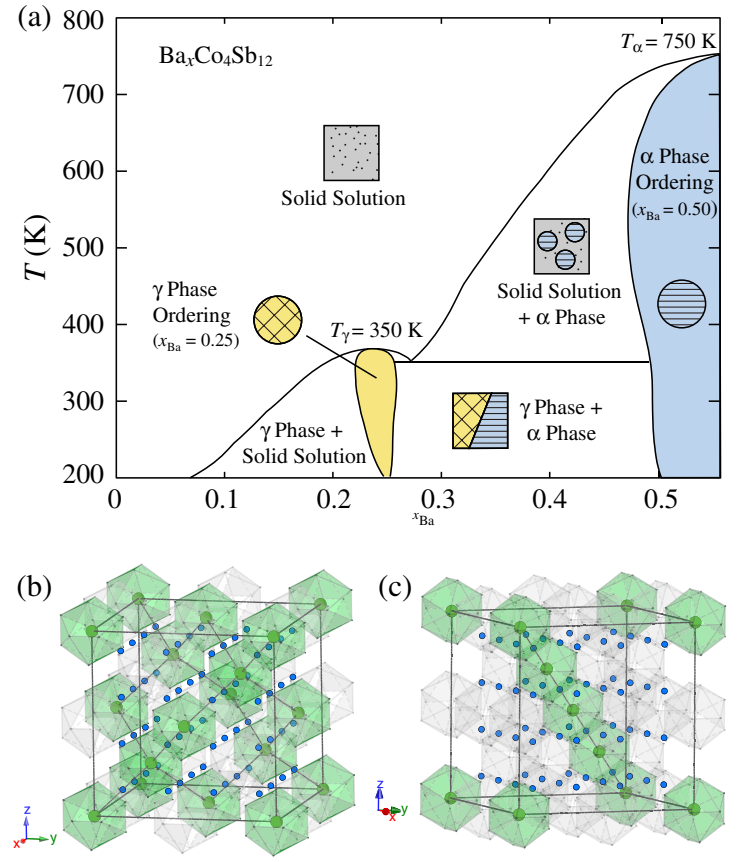

FIG. 1 (color online). (a) The solid-state phase diagram of $\mathrm{Ba}_{x} \mathrm{Co}_{4} \mathrm{Sb}_{12}$ showing the order-disorder transition temperatures $\left(T_{\alpha}\right.$ and $\left.T_{\gamma}\right)$. (b) Atomic structure of $\gamma$ and (c) $\alpha$ phases. The Ba atoms (green circles) are shown in the ordered network structures. The Co atoms are shown with small blue circles. To avoid crowding, the $\mathrm{Sb}$ atoms at the polyhedron vertices are not shown. $\langle 111\rangle$ family axes; it disorders at $T_{\gamma} \approx 350 \mathrm{~K}$. Compared to that, the $\alpha$ phase has a higher symmetry with the filled $\mathrm{Ba}$ sites forming a diamond network; it disorders at $T_{\alpha} \approx 750 \mathrm{~K}$. Because all relevant phase transitions are first order, phase coexistence of the two phases can be obtained at intermediate $x$ and low temperature. At higher temperatures coexistence of each phase with a solid solution is obtained.

The calculation of $\kappa_{L}$, and the interatomic potentials used in the MD simulations for the intermediate concentrations of $\mathrm{Ba}$ were based on the combinative rules (CRs) applied to the empty $\mathrm{CoSb}_{3}$ and fully filled $\mathrm{BaCo}_{4} \mathrm{Sb}_{12}$ potentials from [20]. We use the Morse and three-cosine potentials, parameterized for two-body ( $\mathrm{Co}-\mathrm{Sb}, \mathrm{Co}-\mathrm{Co}$, $\mathrm{Sb}-\mathrm{Sb}$, and $\mathrm{Ba}-\mathrm{Sb}$ ) and three-body (Co-Sb-Sb) interactions $[20,22,28]$. Interatomic potentials are simplified fields and the CRs are approximations, therefore these potentials cannot accurately predict, for example, formation energy. As related to $\kappa_{L}$, the structural parameters $(\mathrm{Co}-\mathrm{Sb}$ first neighbor bond length $r_{\mathrm{o}}$ ), elastic properties (bulk modulus $E$ and elastic constant $C_{i j}$ ), average phonon speeds $u_{p, g, A}$, and specific heat capacity $C_{v}$ (at $300 \mathrm{~K}$ ) found from these potentials and from DFT are summarized in Table I. We compared the acoustic phonon characteristics, predicted using CRs and DFT, and found good agreement in their predicted phonon density of states and its integrated value (Fig. S3 in the supplement [22]). Using CRs, the prediction of $\kappa_{L}$ at intermediate $\mathrm{Ba}$ composition is done with equilibrium MD and the Green-Kubo heat current $(\boldsymbol{q})$ autocorrelation function (HCACF) decay [20,30], i.e.,

$$
\boldsymbol{\kappa}_{L}=\frac{1}{k_{B} T^{2} V} \int_{0}^{\infty}\langle\boldsymbol{q}(t) \boldsymbol{q}(0)\rangle d t, \quad \boldsymbol{q}=\frac{d}{d t} \sum_{i} E_{i} \boldsymbol{r}_{i},
$$

where $k_{B}$ is the Boltzmann constant, $V$ is the MD volume, $T$ is the temperature, $\langle\boldsymbol{q}(t) \boldsymbol{q}(0)\rangle$ is the HCACF tensor, $E_{i}$ is the energy of an atom (excluding the site energy), and $\boldsymbol{r}_{i}$ is the atomic position vector. The details of the MD calculations are given in the supplementary material [22].

From Fig. 1(a), there are six qualitatively different structural configurations possible for Ba concentrations between $0 \leq x \leq 0.5$, at $300 \mathrm{~K}$ : three ordered ground-state structures $(x=0,0.25$, and 0.5$)$, a solid solution $(0<x \leq$ $0.16)$, a mixture of $\gamma$ phase and solid solution $(0.16<x<$ 0.25 ), and a two-phase mixture of ordered phases $(0.25<x<0.5)$. Figure 2(a) shows the predicted $\kappa_{L}$ of $\mathrm{Ba}_{x} \mathrm{Co}_{4} \mathrm{Sb}_{12}$ as a function of $\mathrm{Ba}$ concentrations. As input configurations in the MD simulations, we used the groundstate ordered $\mathrm{Ba}$ configurations at $x=0,0.25$ and $0.5, \mathrm{Ba}$ solid solutions as obtained from MC snapshots $(x=0.03$ and 0.14) and two-phase mixtures with a single interface (e.g., for $x=0.38 \mathrm{MD}$ cell consisted of one half having the $\gamma$ ordering at $x=0.25$ and the other half having $\alpha$ ordering at $x=0.5 ; x=0.33,0.38$, and 0.44 ). The results are in good agreement with experiment [27], showing that as the Ba concentration increases, $\kappa_{L}$ decreases noticeably. 
TABLE I. Comparison of $\mathrm{Ba}_{0.25} \mathrm{Co}_{4} \mathrm{Sb}_{12}$ properties obtained from CRs and DFT. The available results for $\mathrm{CoSb}_{3}$ are also listed. The $u_{p, g, A}$ is along the $\Gamma-\mathrm{N}$ and $\Gamma-\mathrm{H}$ directions.

\begin{tabular}{lcccccc}
\hline \hline & $r_{\mathrm{o}}(\AA)$ & $E(\mathrm{GPa})$ & $C_{11}(\mathrm{GPa})$ & $C_{44}(\mathrm{GPa})$ & $u_{p, g, A}(\mathrm{~m} / \mathrm{s})$ & $C_{v}(\mathrm{~J} / \mathrm{mol}-\mathrm{K})$ \\
\hline CRs & 2.56 & 78 & 155 & 37 & 3098 & 22.9 \\
DFT & 2.54 & 82 & 126 & 54 & 2526 & 23.0 \\
Literature & & $82^{\mathrm{a}}$ & $158^{\mathrm{a}}$ & $57^{\mathrm{a}}$ & $2934^{\mathrm{b}}$ & \\
\hline \hline
\end{tabular}

afrom Reference [29].

${ }^{b}$ from Reference [2].

The minimum conductivity relation $\kappa_{\min }$ for the amorphous phase is also shown, and this gives $\kappa_{\min } \approx 0.37 \mathrm{~W} / \mathrm{m} \mathrm{K}$ using $\mathrm{CoSb}_{3}$ properties [22]. Starting with $\kappa_{L}$ of $\mathrm{CoSb}_{3}$ dominated by phonon-phonon scattering, we consider only phonon-point defects scattering and two-phase scattering for the compounds. On the top of Fig. 2(a), the state of the Ba-vacancy ordering and two-phase mixtures are marked. Since Ba more or less randomly fills the voids of $\mathrm{CoSb}_{3}$ in the solid-solution regime, phonons propagate through the $\mathrm{CoSb}_{3}$ host structure with the randomly distributed $\mathrm{Ba}$ atoms acting as point defects. As the phonon-boundary scattering is negligible except at low temperatures, this can persist up to $x=0.25$ (fully ordered $\gamma$ phase). The overall $\kappa_{L}$ with the inclusion of phonon-point defects scattering is

(a)
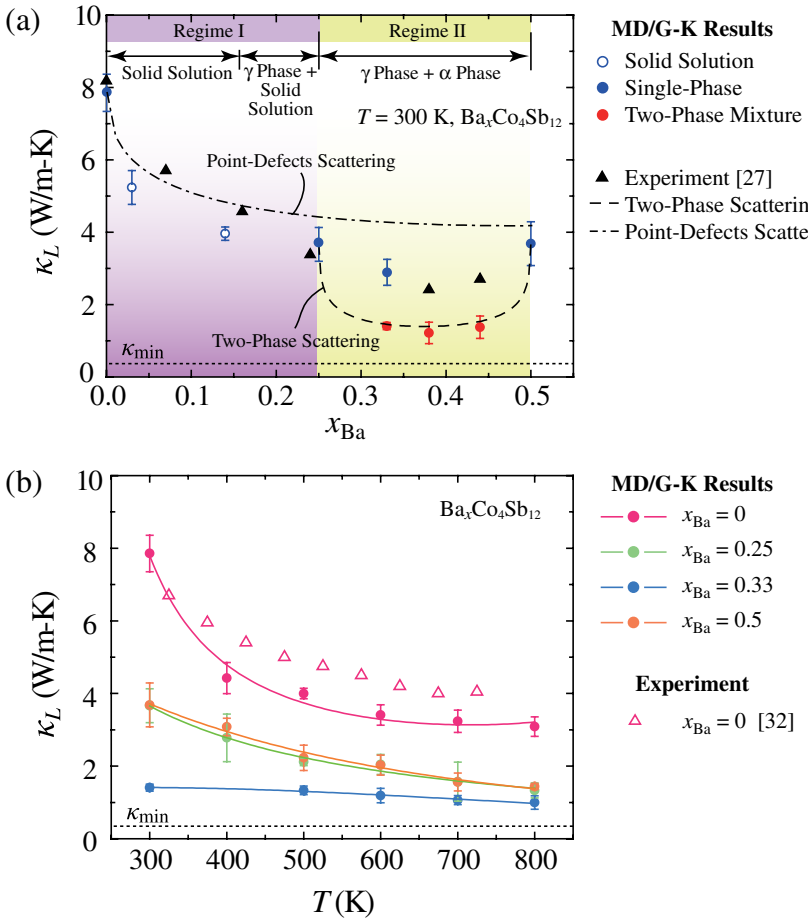

FIG. 2 (color online). Variation of predicted $\kappa_{L}$ of $\mathrm{Ba}_{x} \mathrm{Co}_{4} \mathrm{Sb}_{12}$. (a) Dependency on various $\mathrm{Ba}$ concentration at $300 \mathrm{~K}$. The available experimental results [27], and the results of two analytical models [22] are also shown. (b) Temperature effects for several fill fractions. The available experimental results for $\mathrm{CoSb}_{3}$ [32] are also shown. All error bars represent the standard errors.
$\kappa_{L}(x)=\kappa_{L}(0) /\left\{1+c(x)[x(1-x)]^{1 / 2}\right\}$, where $c(x)$ is the coefficient for point-defects scattering with respect to $x$ [22], and results are shown in Fig. 2(a), predicting significant phonon scattering in regime I. In the two-phase mixture $(0.25<x<0.5)$, the mixture of two ordered phases $(\gamma$ and $\alpha$ ) creates significant interfacial scattering. This overall resistivity includes each phase resistivity $\left(1 / \kappa_{L, \gamma}\right.$ and $\left.1 / \kappa_{L, \alpha}\right)$ and the interfacial resistivity $\left(1 / \kappa_{L, d}\right)$ simultaneously. While isolated point defects scatter phonons due to a point discontinuity in bonding and mass distribution, interfaces between two phases are two-dimensional discontinuities that scatter phonons. This interfacial resistivity had been previously treated the same as the point-defects scattering, so following that we use the treatment of [22], i.e., $1 / \kappa_{L, d}=0.888\left[x_{\gamma}\left(1-x_{\gamma}\right)\right]^{1 / 2}(\mathrm{~m}-\mathrm{K} / \mathrm{W})$. We note that a more appropriate model would include the interface concentration and the impedance mismatch between the two phases as in the phonon-boundary resistance [31]. Then, we have $1 / \kappa_{L}($ two-phase $)=x_{\gamma} / \kappa_{L, \gamma}+\left(1-x_{\gamma}\right) /$ $\kappa_{L, \alpha}+1 / \kappa_{L, d}$, where $x_{\gamma}$ is the fraction of $\gamma$ phase in a $\gamma-\alpha$ mixture. The dash line in Fig. 2(a) is for this two-phase scattering model and is in agreement with MD predictions. We note that it is the two-phase mixture causing the significant reduction in $\kappa_{L}$, by comparing with a hypothetical single-phase and the two-phase structures, for $x=0.33$. The MD results for these structures are $\kappa_{L}$ (single-phase,one of non-ground states) $=2.9$ and $\kappa_{L}($ two-phase $)=1.4 \mathrm{~W} / \mathrm{m}-\mathrm{K}$ [data points and their uncertainties are given in the Fig. 2(a)]. The point-defects and two-phase scattering are two main reasons for the $\kappa_{L}$ reduction in partially filled structures, compared with the empty structure. We also note that in the empty skutterudites the Umklapp scattering is important in the overall thermal conductivity as the point defects and two-phase scattering mechanisms are absent. In Fig. 2(a), the MD predicted $\kappa_{L}(\sim 1.0 \mathrm{~W} / \mathrm{m}-\mathrm{K})$ and the two-phase scattering model show a minimum near $x=0.38$, and this minimum is close to $\kappa_{\min }$, predicted for the amorphous-phase $\kappa_{L}$.

Figure 2(b) shows the predicted $\kappa_{L}$ for $x=0,0.25,0.33$, and 0.5 , as a function of temperature, along with the experimental results [32]. The transition temperatures $\left(T_{\alpha}\right.$ and $\left.T_{\gamma}\right)$ of two ordered phases are marked in Fig. 1(a), and at $T=800 \mathrm{~K}$ there is no ordered structure. So, we use the fully-disordered structures (configuration snapshots of the MC simulations). The single-phase crystal 


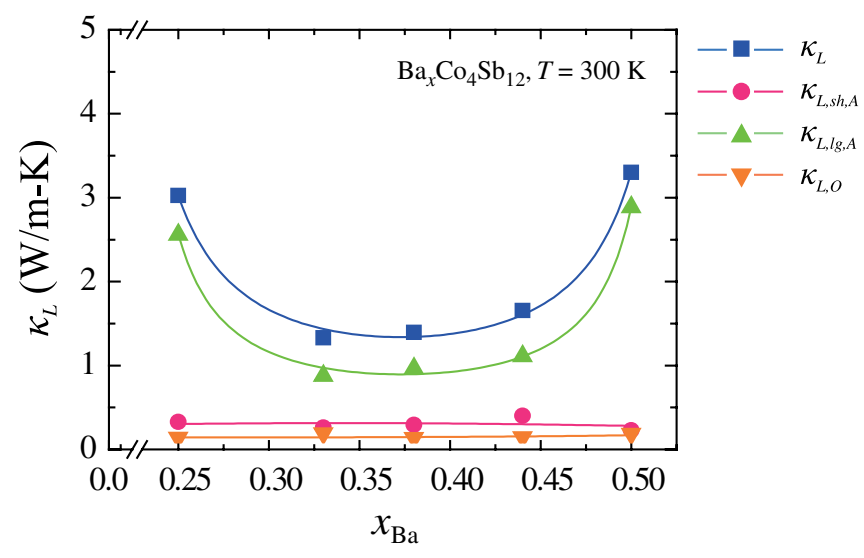

FIG. 3 (color online). $\kappa_{L}$ decomposition and their variations with respect to $\mathrm{Ba}$ concentrations. Decompositions were done using singular MD simulation results for each concentration [as compared to Figs. 2(a) and 2(b) where average over several simulations was used].

follows the Slack relation $\left(\kappa_{L} \sim T^{-1}\right.$ dependence for $T>0.1 T_{D}$ ) [33], while the two-phase mixtures reaches a plateau, similar to amorphous solid $\kappa_{\min }$ [30]. This reconfirms that the two-phase mixtures can be considered as pseudoamorphous structures with significant reduction in $\kappa_{L}$ for such crystalline TE materials.

In general, the heat is carried through skutterudites mostly by acoustic phonons, which are altered by the presence of fillers $[7,18,20,27]$. This significantly reduces $\kappa_{L}$ for the two-phase mixtures and is also confirmed in an analysis of $\kappa_{L}$ decomposition into the short-, long-range acoustic, and optical phonon components [30], i.e.,

$$
\begin{aligned}
\kappa_{L} & =\frac{1}{k_{B} V T^{2}}\left(A_{s h, A} \tau_{s h, A}+A_{l g, A}+\sum_{i} \frac{B_{i, O} \tau_{i, O}}{1+\tau_{i, O}^{2}}\right) \\
& =\kappa_{L, s h, A}+\kappa_{L, l g, A}+\kappa_{L, O},
\end{aligned}
$$

where the $\tau_{i}$ denote time constants, $A_{i}$ and $B_{i}$ are constants, and the subscripts $s h, l g, A$, and $O$ refer to short-range, long-range, acoustic, and optical. After removing the highfrequency components of HCACF with the Fourier lowpass filter $\left(f_{\text {cutoff }}=1.5 \mathrm{THz}\right.$ determined from phonon dispersion curves), the low-frequency acoustic portion are fitted with two exponential decay terms. Each component of HCACF in Eq. (3) was then integrated independently and Fig. 3 shows the results. The long-range acoustic phonon contribution is dominant and most affected by Ba concentrations, while the short-range acoustic and optical components are not. The $\kappa_{L, l g, A}$ decreases most noticeably by the two-phase scattering in the two-phase regime.

Our MD calculations indicate that in order to find a highperformance skutterudite-based TE materials, one can greatly benefit from the presence of order-disorder phase transitions of the filler species in the voids of the skutterudite structure, which leads to two-phase coexistence and the presence of interfaces.

The work is supported by the Center for Solar and Thermal Energy Conversion funded by DOE, Office of Basic Energy Sciences (Grant DESC0000957).

*kaviany@umich.edu

[1] B. C. Sales, D. Mandrus, and R. K. Williams, Science 272, 1325 (1996).

[2] G. P. Meisner et al., Phys. Rev. Lett. 80, 3551 (1998).

[3] G. S. Nolas, J. L. Cohn, and G. A. Slack, Phys. Rev. B 58, 164 (1998)

[4] C. Uher, in Semiconductors and Semimetals, edited by T. Tritt (Academic, San Diego, 2000).

[5] Thermoelectrics Handbook: Macro to Nano, edited by D. M. Rowe (CRC, Boca Raton, 2006).

[6] K. F. Hsu et al., Science 303, 818 (2004).

[7] M. Christensen et al., Nature Mater. 7, 811 (2008).

[8] Y. Kimura and A. Zama, Appl. Phys. Lett. 89, 172110 (2006).

[9] I. Terasaki, Y. Sasago, and K. Uchinokura, Phys. Rev. B 56, R12685 (1997).

[10] J. R. Salvador et al., Philos. Mag. 89, 1517 (2009).

[11] D. T. Morelli and G. P. Meisner, J. Appl. Phys. 77, 3777 (1995).

[12] G. S. Nolas et al., J. Appl. Phys. 79, 4002 (1996).

[13] S. Rundqvist and N. O. Ersson, Arkiv. Kemi 30, 103 (1968).

[14] W. Jeitschko and D. Brown, Acta Crystallogr. Sect. B 33, 3401 (1977).

[15] X. Shi et al., Phys. Rev. Lett. 95, 185503 (2005).

[16] G. A. Slack, in CRC Handbook of Thermoelectrics, edited by D. M. Rowe (CRC, Boca Raton, 1995).

[17] H. Li et al., Appl. Phys. Lett. 94, 102114 (2009).

[18] M. M. Koza et al., Nature Mater. 7, 805 (2008).

[19] D. Wee et al., Phys. Rev. B 81, 045204 (2010).

[20] B. L. Huang and M. Kaviany, Acta Mater. 58, 4516 (2010).

[21] J. M. Sanchez, F. Ducastelle, and D. Gratias, Physica (Amsterdam) 128A, 334 (1984).

[22] See supplementary material at http://link.aps.org/ supplemental/10.1103/PhysRevLett.105.265901.

[23] G. Kresse and J. Furthmüller, Phys. Rev. B 54, 11169 (1996).

[24] J. P. Perdew, K. Burke, and M. Ernzerhof, Phys. Rev. Lett. 77, 3865 (1996).

[25] P. E. Blöchl, Phys. Rev. B 50, 17953 (1994).

[26] G. Kresse and D. Joubert, Phys. Rev. B 59, 1758 (1999).

[27] L. D. Chen et al., J. Appl. Phys. 90, 1864 (2001).

[28] M. Marathe, M. Imam, and S. Narasimhan, Phys. Rev. B 79, 085413 (2009).

[29] L. Zhang et al., Mater. Sci. Eng. B 170, 26 (2010).

[30] M. Kaviany, Heat Transfer Physics (Cambridge, New York, 2008).

[31] S. Shin et al., Phys. Rev. B 82, 081302(R) (2010).

[32] Y. Kawaharada et al., J. Alloys Compd. 315, 193 (2001).

[33] G. A. Slack, in Solid State Physics, edited by F. Seitz, D. Turnbull, and H. Ehrenreich (Academic, New York, 1979) 